\title{
On the Representation of Natural Numbers in Positional Numeral Systems ${ }^{1}$
}

\author{
Adam Naumowicz \\ Institute of Computer Science \\ University of Białystok \\ Akademicka 2, 15-267 Białystok, Poland
}

\begin{abstract}
Summary. In this paper we show that every natural number can be uniquely represented as a base- $b$ numeral. The formalization is based on the proof presented in [11]. We also prove selected divisibility criteria in the base-10 numeral system.
\end{abstract}

MML identifier: NUMERAL1, version: 7.8.03 4.76.959

The notation and terminology used in this paper have been introduced in the following articles: [13], [15], [2], [1], [17], [12], [14], [6], [4], [5], [8], [9], [10], [16], [7], and [3].

\section{Preliminaries}

One can prove the following propositions:

(1) For all finite 0 -sequences $d, e$ of $\mathbb{N}$ holds $\sum\left(d^{\frown} e\right)=\sum d+\sum e$.

(2) Let $S$ be a sequence of real numbers, $d$ be a finite 0 -sequence of $\mathbb{N}$, and $n$ be a natural number. If $d=S \uparrow(n+1)$, then $\sum d=\left(\sum_{\alpha=0}^{\kappa} S(\alpha)\right)_{\kappa \in \mathbb{N}}(n)$.

(3) For all natural numbers $k, l, m$ holds $\left(k\left(l^{\kappa}\right)_{\kappa \in \mathbb{N}}\right)\lceil m$ is a finite 0 -sequence of $\mathbb{N}$.

(4) Let $d, e$ be finite 0 -sequences of $\mathbb{N}$. Suppose len $d \geq 1$ and len $d=$ len $e$ and for every natural number $i$ such that $i \in \operatorname{dom} d$ holds $d(i) \leq e(i)$. Then $\sum d \leq \sum e$.

\footnotetext{
${ }^{1}$ This work has been partially supported by the FP6 IST grant TYPES No. 510996.
} 
(5) Let $d$ be a finite 0 -sequence of $\mathbb{N}$ and $n$ be a natural number. If for every natural number $i$ such that $i \in \operatorname{dom} d$ holds $n \mid d(i)$, then $n \mid \sum d$.

(6) Let $d, e$ be finite 0 -sequences of $\mathbb{N}$ and $n$ be a natural number. Suppose $\operatorname{dom} d=\operatorname{dom} e$ and for every natural number $i$ such that $i \in \operatorname{dom} d$ holds $e(i)=d(i) \bmod n$. Then $\sum d \bmod n=\sum e \bmod n$.

\section{Representation of Numbers in the Base- $b$ Numeral System}

Let $d$ be a finite 0 -sequence of $\mathbb{N}$ and let $b$ be a natural number. The functor value $(d, b)$ yields a natural number and is defined by the condition (Def. 1).

(Def. 1) There exists a finite 0 -sequence $d^{\prime}$ of $\mathbb{N}$ such that $\operatorname{dom} d^{\prime}=\operatorname{dom} d$ and for every natural number $i$ such that $i \in \operatorname{dom} d^{\prime}$ holds $d^{\prime}(i)=d(i) \cdot b^{i}$ and value $(d, b)=\sum d^{\prime}$.

Let $n, b$ be natural numbers. Let us assume that $b>1$. The functor $\operatorname{digits}(n, b)$ yields a finite 0 -sequence of $\mathbb{N}$ and is defined as follows:

(Def. 2)(i) value $(\operatorname{digits}(n, b), b)=n$ and $(\operatorname{digits}(n, b))(\operatorname{len} \operatorname{digits}(n, b)-1) \neq 0$ and for every natural number $i$ such that $i \in \operatorname{dom} \operatorname{digits}(n, b)$ holds $0 \leq$ $(\operatorname{digits}(n, b))(i)$ and $(\operatorname{digits}(n, b))(i)<b$ if $n \neq 0$,

(ii) $\operatorname{digits}(n, b)=\langle 0\rangle$, otherwise.

One can prove the following two propositions:

(7) For all natural numbers $n, b$ such that $b>1$ holds len $\operatorname{digits}(n, b) \geq 1$.

(8) For all natural numbers $n, b$ such that $b>1$ holds value $(\operatorname{digits}(n, b), b)=$ $n$.

\section{Selected Divisibility Criteria}

One can prove the following propositions:

(9) For all natural numbers $n, k$ such that $k=10^{n}-1$ holds $9 \mid k$.

(10) For all natural numbers $n, b$ such that $b>1$ holds $b \mid n$ iff $(\operatorname{digits}(n, b))(0)=0$.

(11) For every natural number $n$ holds $2 \mid n$ iff $2 \mid(\operatorname{digits}(n, 10))(0)$.

(12) For every natural number $n$ holds $3 \mid n$ iff $3 \mid \sum \operatorname{digits}(n, 10)$.

(13) For every natural number $n$ holds $5 \mid n$ iff $5 \mid(\operatorname{digits}(n, 10))(0)$.

\section{REFERENCES}

[1] Grzegorz Bancerek. The ordinal numbers. Formalized Mathematics, 1(1):91-96, 1990.

[2] Czesław Byliński. Functions and their basic properties. Formalized Mathematics, 1(1):5565, 1990.

[3] Czesław Byliński. Functions from a set to a set. Formalized Mathematics, 1(1):153-164, 1990. 
[4] Jarosław Kotowicz. Real sequences and basic operations on them. Formalized Mathematics, 1(2):269-272, 1990.

[5] Rafał Kwiatek. Factorial and Newton coefficients. Formalized Mathematics, 1(5):887-890, 1990.

[6] Library Committee of the Association of Mizar Users. Binary operations on numbers. To appear in Formalized Mathematics.

[7] Karol Pąk. Stirling numbers of the second kind. Formalized Mathematics, 13(2):337-345, 2005.

[8] Konrad Raczkowski. Integer and rational exponents. Formalized Mathematics, 2(1):125$130,1991$.

[9] Konrad Raczkowski and Andrzej Nȩdzusiak. Real exponents and logarithms. Formalized Mathematics, 2(2):213-216, 1991.

[10] Konrad Raczkowski and Andrzej Nȩdzusiak. Series. Formalized Mathematics, 2(4):449452, 1991.

[11] Wacław Sierpiński. Elementary Theory of Numbers. PWN, Warsaw, 1964.

[12] Andrzej Trybulec. Subsets of complex numbers. To appear in Formalized Mathematics.

[13] Andrzej Trybulec. Tarski Grothendieck set theory. Formalized Mathematics, 1(1):9-11, 1990.

[14] Michał J. Trybulec. Integers. Formalized Mathematics, 1(3):501-505, 1990.

[15] Zinaida Trybulec. Properties of subsets. Formalized Mathematics, 1(1):67-71, 1990.

[16] Tetsuya Tsunetou, Grzegorz Bancerek, and Yatsuka Nakamura. Zero-based finite sequences. Formalized Mathematics, 9(4):825-829, 2001.

[17] Edmund Woronowicz. Relations defined on sets. Formalized Mathematics, 1(1):181-186, 1990.

Received December 31, 2006 